\title{
Prediction and evaluation of photovoltaic-thermal energy systems production using artificial neural network and experimental dataset
}

\author{
Jabar H. Yousif*, Hussein A. Kazem \\ Faculty of Computing and Information Technology, Sohar University, PO Box 44, Sohar, PCI 311, Oman
}

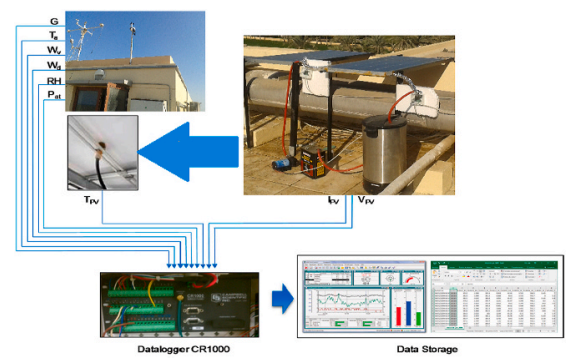

\section{A R T I C L E I N F O}

\section{Keywords:}

Solar energy

Photovoltaic performance

Hybrid PV/T

Energy prediction

ANN

\begin{abstract}
A B S T R A C T
Photovoltaic/thermal (PV/T) systems combine two collectors, which increase efficiency, reduce cost and space, and produce electricity and heat, simultaneously. Many factors affect PV/T current, voltage, power, efficiency, and heat energy production. For example, the location of the PV system, ambient temperature, irradiance, humidity, dust, and many other factors. Also, different modelling techniques are used to evaluate PV/T efficiency, for example, analytical, regression, numerical, artificial neural network (ANN). The current work aims to predict and assess a PV/T system using ANN models based on an experimental dataset in Oman. The PV/T system with weather station and data acquisition was installed in Sohar, Oman. The weather and electrical data has been recorded. A novel mathematical and ANN model for examining the performance of $\mathrm{PV} / \mathrm{T}$ systems has been developed. The experimental results show improvement in PVT power production $(68.6132 \mathrm{~W}$ ) compared to the conventional PV $(66.7827 \mathrm{~W})$. The results demonstrate that the three proposed models (MLP, SOFM, and SVM) achieved excellent MSE results for generating the current values of the PV system $(0.00043,0.00030,0.00041)$ and PV/T system
\end{abstract}

\footnotetext{
* Corresponding author. PH: +968-95030520.

E-mail address: jyousif@soharuni.edu.om (J.H. Yousif).
} 
(0.00719, 0.00683, 0.00763), respectively. Also, the proposed models delivered excellent MSE results for simulating the power values of the PV system $(0.04457,0.05006,0.13816)$ and PV/T system $(0.04457,0.05006,0.13816)$, respectively. The proposed models result validated with experimental data using descriptive statistics and Evaluation Metrics. Finally, the proposed neural models can generate future figures for any needed period that accurately fit the actual datasets.

\section{Introduction}

Solar energy is an essential energy source, which utilizes light and heat and can be employed using photovoltaic, and thermal energy systems. Also, the thermal collector could be connected to the back PV module to produce a hybrid PV/T system. Chaichan et al. [1] found that PV modules can convert light to electricity with about $20 \%$ of the received solar energy. Most of the energy not transferred to electricity is converted to heat. Hybrid PV/T systems convert solar irradiance into thermal and electrical energy at the same time [2]. PV/T technology includes different configurations and products based on the use of PV/T collectors, such as Air-based, Liquid-based, Water cooling, Refrigerant cooling, Nanofluid cooling, Bi-fluid, heat pump, and concentrator.

Solar energy is primarily collected in one of two ways, either directly by photovoltaic cells (PV), or by collecting solar thermal energy by pipes, dishes, mirrors, or lenses to produce heat, which is used to heat air, fluids, buildings, etc. [3]. PV converts solar irradiance into a direct electrical current, which is usually a manufactured semiconductor made from silicon. In practice, the PV cell has a thickness, and a surface of $0.25-0.35 \mathrm{~mm}$ and $100-225 \mathrm{~m}^{2}$, respectively [4]. There are different types of PV, such as mono-crystalline silicon, multi-crystalline silicon, amorphous silicon, and organic solar cells [5].

Many factors affect the production of PV/T current, voltage, power, efficiency, and heat energy production. For example, the location of the PV system, ambient temperature, irradiance, dust, humidity, rainfall, and many other factors [6]. Different factors are considered in PV/T design, model, and evaluation, such as modelling techniques used to evaluate PV/T, for example, the analytical, regression, numerical, artificial neural network (ANN) [7].

ANN is defined as a mathematical algorithm that simulates the human brain functions, such as learning and training [8]. The ANN networks depend on the input of real data and the used pre-specified function, which is to be set by the programmer, to produce the desired output results. The ANN is a complex adaptive system that could change its internal structure based on the input information to improve adaptability and performance. ANN has become increasingly helpful for system modeling and the optimization of results. ANN computation has augmented our ability to process, and analyze data. ANN is a strong modeling tool, which maps a complex input into a simple output space [9]. In general, ANN applications tend towards the classification, recognition, generalization, prediction, and organization of data. The network architecture determines the topology of the connections between neurons (input, hidden, and output). Many researchers used ANN to model and evaluate PV and PV/T systems [10].

Saberian et al. [11] proposed a prediction model of photovoltaic at "King Abdulaziz City for Science and Technology" in Saudi Arabia located at the location of (longitude $42.661 \mathrm{~W}$ and latitude 18.231 N). The collected data has been trained using a feedforward artificial neural network (FFANN) testing 3 different input data. The first one using the maximum temperature in neuron networks that have 2 inputs, 24 hidden layers and 1 output, getting a mean squared error (MSE) $=2.823 \times 10^{-4}$, mean absolute percentage error $(\mathrm{MAPE})=10.3 \%$. The second one uses the mean temperature in neuron networks that have 2 inputs, 32 hidden layers and 1 output getting MSE $=0.0052$, MAPE $=111.8 \%$. The third one uses the average relative humidity and temperature in neuron networks with 3 inputs, 24 hidden layers, and 1 output getting MSE $=0.0003$, MAPE $=4.49 \%$, which led to the conclusion that ANN can estimate global solar irradiance (GSR).

Ghanbarzadeh et al. [12] and Behrang et al. [13] conducted a study in Drzul-Iran (32.16 N and 48.25 E) which investigated three cases from measured data in 2002-2005 to predict GSR. The first case used relative humidity, daily mean air temperature, and length of day as inputs where MAPE and MSE were found to be $14.5 \%$, and 0.008879 , respectively. In case 2 , the inputs are sunshine hours, daily mean air temperature, and length of the day, which provides MAPE and MSE results of 9.4\%, and 0.0043. In the last case where relative humidity, daily mean air temperature, and sunshine hours are required, best results were attained where MAPE and MSE are determined to be $8.84 \%$, and 0.0040 , respectively. Results obtained indicate that ANN can be a trusted method for estimating GSR.

Rehman et al. studied two models, one for estimating solar radiation intensity ratio $\mathrm{H} / \mathrm{H}_{0}$ and the other for modeling $\mathrm{H}$ to be output. The input parameters are $\mathrm{H}_{0}, \mathrm{~T}_{\max }, \mathrm{T}_{\min }$, and $\mathrm{H}$. The statistical errors used are NMSE, NRMSE, $\mathrm{R}^{2}$, RMSE, MSE, MAE, and MBE. The data collected from Almeria in Spain, and the results, give greater accuracy than conventional regressions models [14].

Mellit et al. [15] conducted a study in South India (Madurai, which is located at 78.1198E and 9.9252 N). Seven variables have been investigated, which are yearly and monthly humidity, wind speed, atmospheric and air temperature (daily average values). ANN was used to train and test the variables using "evenberg-marquardt LM", "batch gradient descent GD", "resilient backpropagation RP", and "scaled conjugate gradient SCG" algorithms. The inputs, neurons, hidden and output used to construct the ANN, are 7, 15, 1, and 1 respectively, to predict GSR, by determining R, RMSE, and MAE. The best result was performed using LM algorithm with MAE = 3.3639 , $\mathrm{RMSE}=4.8372$, and $\mathrm{R}=0.9994$ for the testing result, and $\mathrm{MAE}=1.7212$, $\mathrm{RMSE}=2.0028$, and $\mathrm{R}=0.9996$ for training values.

Asl et al. [16] from Dezful in Iran (located at 32.1648 N, and 48.25E) proposed an ANN model based on measured parameters. The measurement was conducted for the relative humidity, the daily mean air temperature, the evaporation, the soil temperature, day of the year, sunshine hours, and wind speed at the duration between 2002 and 2005. The data used to train the ANN using MLP, and for 214 days in 2006 to be tested. The MLP consists of 3 neurons in the first hidden layer and 2 in the second, with the sigmoid transferred function used for hidden layers and for the output layer, a linear transferred, LM algorithm gave the best prediction. Using MPL testing 
data gives MAPE $=6.08 \%$, and $\mathrm{R}^{2}=99.03 \%$ and training data performed MSE $=0.0042$, and SEE $=5.9278$, which is acceptable for estimating GSR.

Al-Shamisi et al. [17] provides research from UAE AL-Ain city (located at altitude 24.1302 N and longitude 55.8023E) using ANN MLP and RBF techniques. The input parameters used are sunshine hours, maximum temperature, mean relative humidity, and mean wind speed. The proposed ANN model was evaluated using $\mathrm{R}^{2}$, RMSE, and MBE. The samples of input data are collected for 10 years (1995-2004) used to train the network. Data for 3 years (2005-2007) are used for testing the proposed models. The results show that the RBF technique is superior to the MPL in most cases.

Qin et al. [18] made a comparison between simulated GSR and the observed one in the Tibetan Plateau and the nearby area in China. The study covers the area from 25 to $45 \mathrm{~N}$ and from 70 to 105E using the ANN-MLP method, with statistical parameters RMSE, and R. It was observed that ANN provides high accuracy, and best stability compared with JAXA.

Hasni et al. [19] tested the ANN model for estimating GSR in Algeria's southwestern Drylands. The data was collected for 5 months and tested by ANN LM feedforward backpropagation algorithm, with a hidden layer of 3 neurons. The proposed model has an RMSE of 0.084 , MAE of 2.9999, and $\mathrm{R}^{2}$ of 0.9998 .

Khatib et al. [20] used the MLP ANN network to train, test, and predict GSR in five cities in Malaysia. The results found the Bharu Kuching station to be more accurate compared with other investigated stations. However, the Alor Setar station was found to be the least accurate model, with $0.78 \% \mathrm{MBE}$ and 7.6\% RMSE for the best case, and 3.45\% MBE and 9\% RMSE for the worst case. The researchers confirm that GSR prediction is more accurate using ANN models.

Salima et al. [21] conducted a study in Malawi which was held for six metrological stations. It is noted that fairly agreed between measured values and calculated values for solar irradiance at the six stations was presented. Ben et al. [22] present a study that shows an algorithm that determines the OPOP of the PV/T function of the variation of solar irradiance and ambient temperature. The result confirms that the calculated values by ANN are fast and accurate.

Assi et al. [23] proposed RBF models to predict GSR in UAE. Data for the duration from, 2002 to 2008 was used to train the model, whereas data between the duration from 2009 to 2010 are used to test and validate the prediction model. The result showed that all models are performed well with $\mathrm{R}^{2}$ above $82 \%$.

ANN model developed for estimating monthly mean GSR in eight cities in China using FFBPA to measure data on MPE, MBE, and RMSE [24]. In Al-Madinah, Saudi Arabia, a comparative study was made between BNN and empirical models to estimate GSR [25]. Data has been collected for three cities (New Delhi, Hyderabad, and Mumbai in India) for ninety days. The data are used for testing the network with indicators such as RMSE, MAPE, and MBE. It was found that MAPE is minimum when considering temperature, month, date, and humidity as inputs [26]. Two proposed models from Qena and Egypt have been proposed to estimate GSR, which show a close result between estimated and measured values of 0.998 for correlation coefficient, $48 \mathrm{Wh} / \mathrm{m}^{2}$ for MBE, and $115 \mathrm{Wh} / \mathrm{m}^{2}$ as RMBE $[27,28]$. A predicted data output power is used for the PV model, which shows the relativity between the training sample and predicted data. The predicted power data shows that the method used gives high predicted accuracy suitable for engineering applications. In Amman Jordan, three ANN models are used, FFN, Elman, and NARX to predict the solar irradiance using MATLAB software, and metrological data as inputs. The result indicates that NARX has a good ability to predict solar irradiance [29]. In Ghardaia Algeria the radiometric station parameters are used as an input to ANN to estimate GSI evaluating statistical formulas such as RMSE, MBE, and ${ }^{2}$.

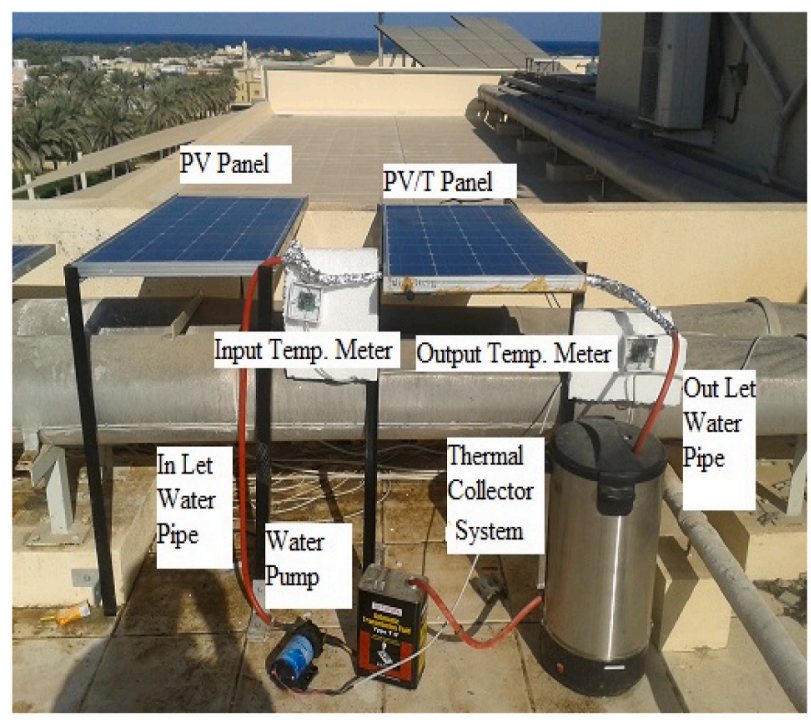

(a)

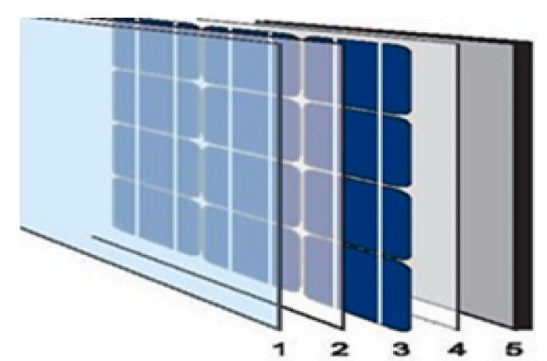

(b)

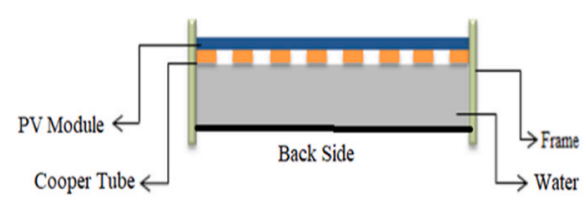

(c)

Fig. 1. (a) PV/T systems experimental setup; (b) Solar module constructions, (1) tempered glass, (2) ethylene Vinyl Acetate EVA, (3) cell, (4) Ethylene Vinyl Acetate EVA, (5) Tedlar Polyester Tedlar TPT; (c) cross-section of PV Panel. 
The result shows that ANN gave the best performance when compared with empirical models [30]. The result shows that ANN gave the best performance when compared with empirical models [30]. In Abu Dhabi, UAE, metrological data for 10 years were used to train MLP and RBF as [31] built-in integrated PV/T, which approximates the PV integrated model under fixed conditions. The result indicates that ANN can learn the underlying relationships between input and output data for that application. The trained ANN can be a good tool for building integrated PV/T arrays in selecting optimal information. Ahmed et al. [32] used the ANN LM model to investigate solar irradiance. Comparing it with validation data, using MSE, and R values, six models were proposed and tested, the best one giving 0.0591, and 0.963 for MSE, and R respectively. Saberian et al. [11] used GRNN and FFBP methods for training metrological data and estimated power derived from mathematical equations, the result shows that both neural networks have good modelling performance.

The current work aims to predict and assess a photovoltaic-thermal energy system using artificial neural network models based on the experimental dataset in Sohar/Oman. In addition, the proposed neural models can generate future figures for any needed period that accurately fit the actual datasets. The contribution of this study is highlighted as follows:

- Develop novel mathematical and ANN models for examining the performance of PV/T systems.

- Compare the mathematical forecasting models with the proposed models and the experimental results.

- The electrical power/efficiency of the proposed PV/T models to be compare with different literature accordingly.

- Validating the results with experimental data using descriptive statistics and Evaluation Metrics.

\section{Experimental setup}

\subsection{The used $P V / T$ system}

Fig. 1(a) illustrates the experimental PV/T system setup, which contains two PV panels located on the surface roof of the faculty of engineers in Sohar University. These monocrystalline silicon solar panels have the following specifications: open-circuit voltage $V_{O C}=$ $22.32 \mathrm{~V}$, short-circuit current $I_{C S}=5.94 \mathrm{~A}$, and rated power $P_{\text {rated }}=100 \mathrm{~W}$.

As shown in Fig. 1(a), $2 \mathrm{~m}$ of the copper tube are fixed at the input and the output, to read the water temperature. These meters were W1209 digital temperature controllers and the temperature value is displayed in ${ }^{\circ} \mathrm{C}$ via 3-digit seven-segment displayers. A $24 \mathrm{~V}$ water pump is used to circulate the water, with a $1 \mathrm{~A}$ and $1 \mathrm{~L}$ per minute water flow rate. The hot water is pumped from the panel to the thermal collector system. It is worth mentioning that the pump power consumption is not considered in this study. The study monitored the temperature for three days from the 16th - 18th October 2020 from 09:00 a.m. to 04:00 p.m. Data Acquisition DAQ process is used to measure the panel's voltage and current, the ambient temperature, and the irradiance. DAQ consists of sensors and measurement hardware that acts as an interface between the computer and the signals from outside, and a computer with programmed software which controls the operation of the DAQ device is used for processing, storming, and visualizing the measured data.

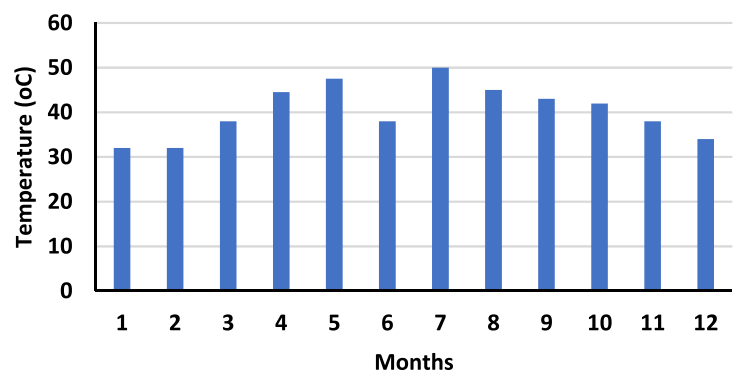

(a)

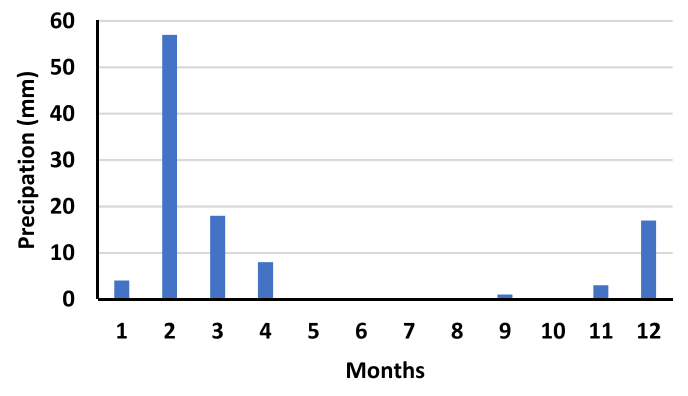

(b)

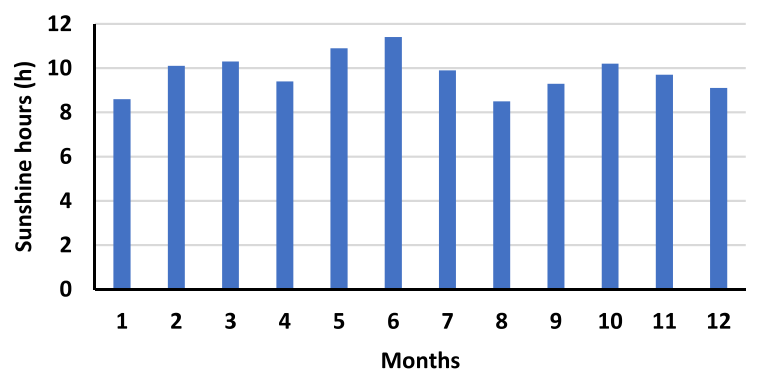

(c)

Fig. 2. (a) Average temperature per month; (b) Rainfalls in Sohar; (c) Sunshine Hours in Sohar. 
Fig. 1(b) describes the five layers that the panel is constructed from. The five layers are: Layer 1 is tempered glass it adds strength to the panel and prevents light being reflected because it is coated, Layer 2 is Ethylene Vinyl Acetate (EVA) and Tedlar Polyester Tedlar (TPT) to protect the cell from water and from being destroyed, Layer 3 are the cells, Layer 4 is the EVA layer and Layer 5 the TPT layer. In Fig. 1(a) two modules are used for this study, one with a thermal collector and the other without. The backside of the thermal collector panel is closed, and a folded copper tube is fixed inside which is used to collect the heat from the panel as shown in Fig. 1(c). Also, this volume is filled with water to improve the cooling system.

\subsection{Sohar metrological data}

Sohar city is located in Oman's northern coast $24.3461 \mathrm{~N}, 56.7075 \mathrm{E}$, and is $12 \mathrm{~m}$ above average sea level. It has a very hot desert climate especially during summers and mild winters. Fig. 2(a) shows average temperature measured for 12 months in 2019 , where in July it reached its highest value of $50{ }^{\circ} \mathrm{C}$. With very low precipitation, most of the rainfall occurs during February, shown in Fig. 2(b). Another important factor in photovoltaic performance is the sunshine hours, displayed in Fig. 2(c) [33,34].

The total amount of solar irradiance received by the earth service is called Global Solar Radiation (GSR) calculated in watts per meter square; it is the sum of the reflected and direct diffuse solar radiation. GSR has been the focus of many studies because it provides energy amounts according to climate stations. In order to measure solar irradiance, expensive devices are needed to be installed and maintained. ANN method is an excellent method to estimate global solar radiation, and it is helpful for solar system designers to have trusted information about solar irradiance. The information can be arranged in tabular form or map form within the zone that the installation will be made. Data collected during one month in 15 min' intervals, and the best 3 days' records were accepted as representative samples. According to weather conditions, input and output water temperature in the PV/T panel are recorded manually, and voltage, current, power, ambient temperature, and irradiance, are stored by the Data Acquisition System as shown in Fig. 3.

\subsection{Performance evaluation criteria}

Different methods were used to compare ANN models' performance, such as MSE (Mean Squares Error), MAE (Mean Absolute Errors), NMSE (normalized mean square error), and R-square (coefficient of determination).

The MSE is computed the two times average cost as in equation (1).

$$
M S E=\frac{\sum_{j=o}^{P} \sum_{i=0}^{N}\left(d_{i j}-y_{i j}\right)^{2}}{N P} .
$$

where $P$ is the entirety processing item, $N$ is the entirety exemplars. The $y_{i j}$ is the current output of item (i). The $d_{i j}$ is the experimental desired output for the item (i).

The NMSE measures the distance between estimated and actual outcomes, which defines as in equation (2):

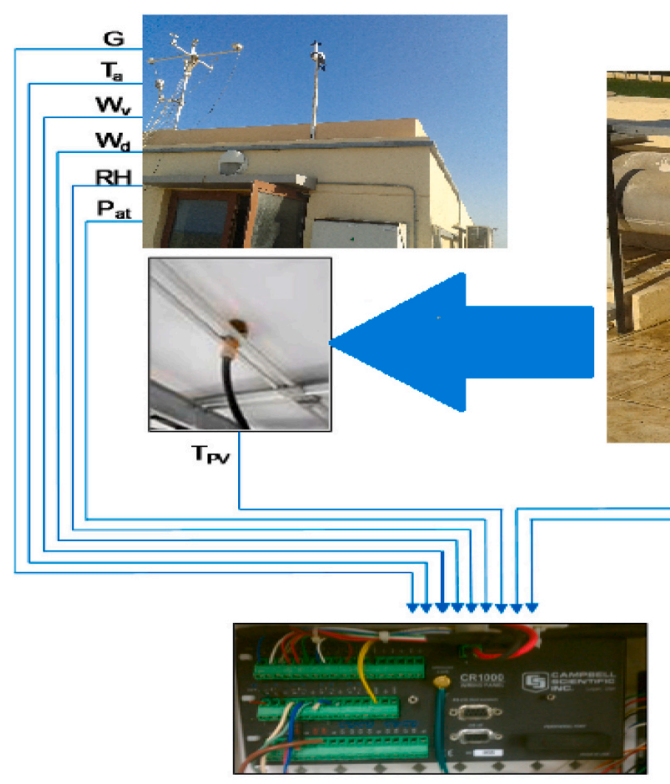

Datalogger CR1000
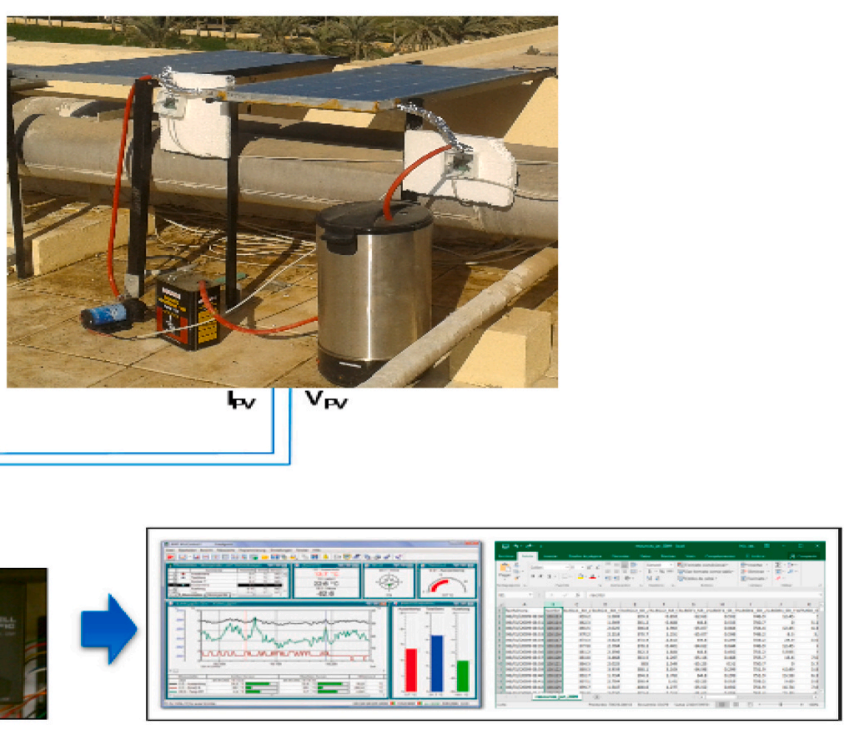

Data Storage

Fig. 3. Experiment setup and process. 


$$
N M S E=\frac{P N M S E}{\sum_{j=o}^{P} \frac{N \sum_{i=0}^{N} d_{i j}^{2}-\left(\sum_{i=0}^{N} d_{i j}\right)^{2}}{N}}
$$

The MAE determines the Average mean absolute error between the predicted and experimental desired values. The lower value of MAE indicates a better, long-term model prediction.

It defines as in equation (3).

$$
M A E=\frac{1}{n} \sum_{i=1}^{n}\left|p r_{i}-d_{i}\right|
$$

where $\mathrm{pr}_{\mathrm{i}}$ is the predicted value, and $\mathrm{di}$ is the desired value.

R-Square indicates how the data are close to the fitted regression line; its value is in the range $(0,1)$. Zero indicates none of the data's variables is around the standard mean, and 1 explains that all variables are around the standard mean. It is describing in equation (4).

$$
\mathrm{R}-\text { Square }=1-\frac{\sum_{i=1}^{n}\left(d_{i}-p r_{i}\right)}{\sum_{i=1}^{n}\left(d_{i}-\overline{\bar{d}}_{i}\right)}
$$

The $\overline{\bar{d}}$ is the mean of the values in $\mathrm{d}$.

\subsection{ANN methods}

The new development of artificial neural networks (ANN) helps deploy linear or nonlinear associations and reduces data dimensionality. NN is an inspired computational model that emulates human brain activities and imitates intelligent responses. The NN has unique features that include parallelism and recurrent computing, making it a cable of learning from previous experience [35]. Therefore, it is appropriate for many applications, such as data classification and recognition, information processing and prediction of future figures, etc. Numerous NN architectures were used to monitor and evaluate the performance of renewable generation systems.

Many factors affect the choice of neural networks (NN), which depends on the type of application and the size and type of data available. Therefore, it is not easy to choose a specific NN type, so what can be applied to a data type has the opposite results with other data in another region. Hence, forecasting future data helps to give a clear idea of the data and the requirements that must be met or the precautions required to be taken.

The NN consists of one layer or multilayers that the first layer uses to input data and the last layer for output the result. Any inbetween layers are called hidden layers, as shown in Fig. 4(a).

\subsubsection{Multilayer perceptron (MLP)}

MLP is a supervised learning technique deployed in classification and prediction tasks. MLP topology consists of multi-layered neurons including input, hidden, and output layers, as shown in Fig. 4(a). The MLP network is used for analyzing the behavior of experimental solar data and predicting future figures.

The backpropagation learning technique computes the errors through the network and allows adjustment of the hidden layers' weights, which is defined in equation (5):

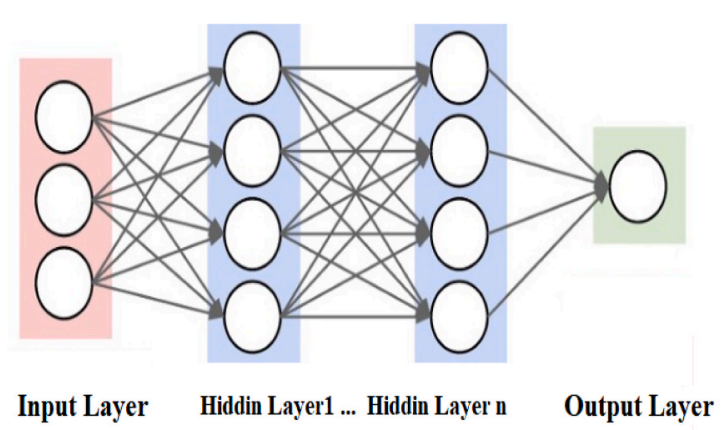

(a)

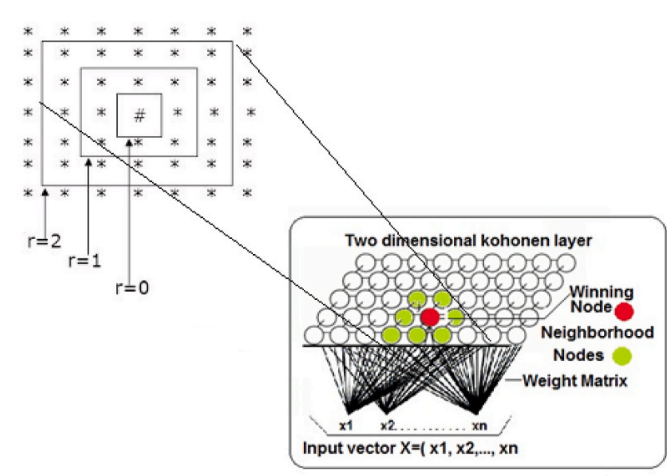

(b)

Fig. 4. ANN architecture (a) MLP; (b) SOFM. 


$$
e_{i}(n)=d_{i}(n)-y_{i}(n)
$$

where di is the desired dataset, and yi is the computed data value.

The evolution of the cost function gradient between the next iteration is slow, which drives a slow convergence. Therefore, the momentum learning technique is used to overcome this problem, which will smooth the behavior and speed up the convergence. The momentum learning method is computed in equation (6):

$$
w_{i j}(n+1)=w_{i j}(n)+\eta \delta_{i}(n)+x_{j}(n)+\alpha\left(w_{i j}(n)-w_{i j}(n-1)\right)
$$

where $\alpha$ is the momentum factor, which is typically set to $0.7 . \eta$ is the constant step size, And $\delta_{i}(n)$ is the local error.

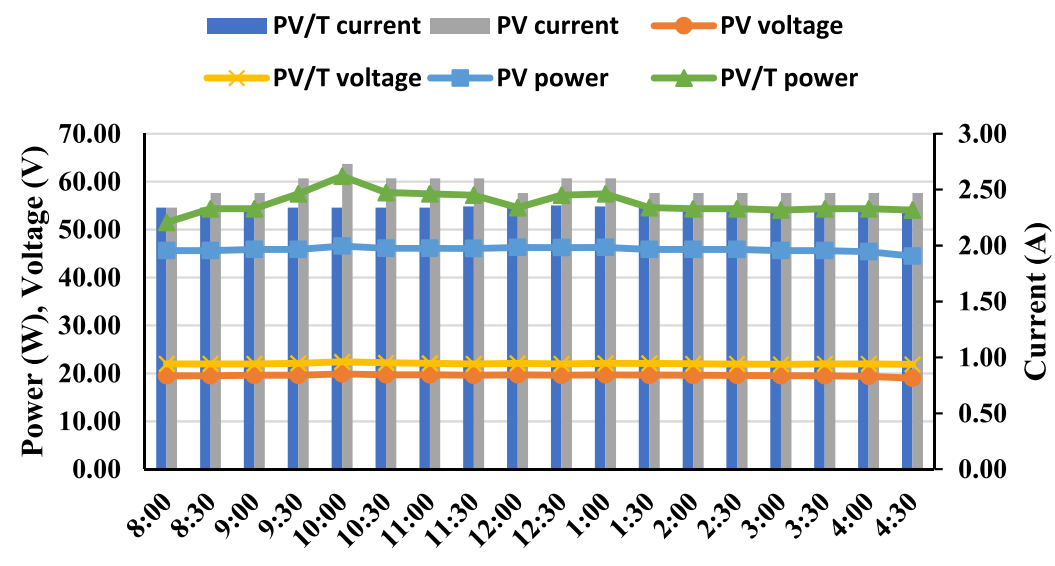

Time (Hour:Minute)

(a)

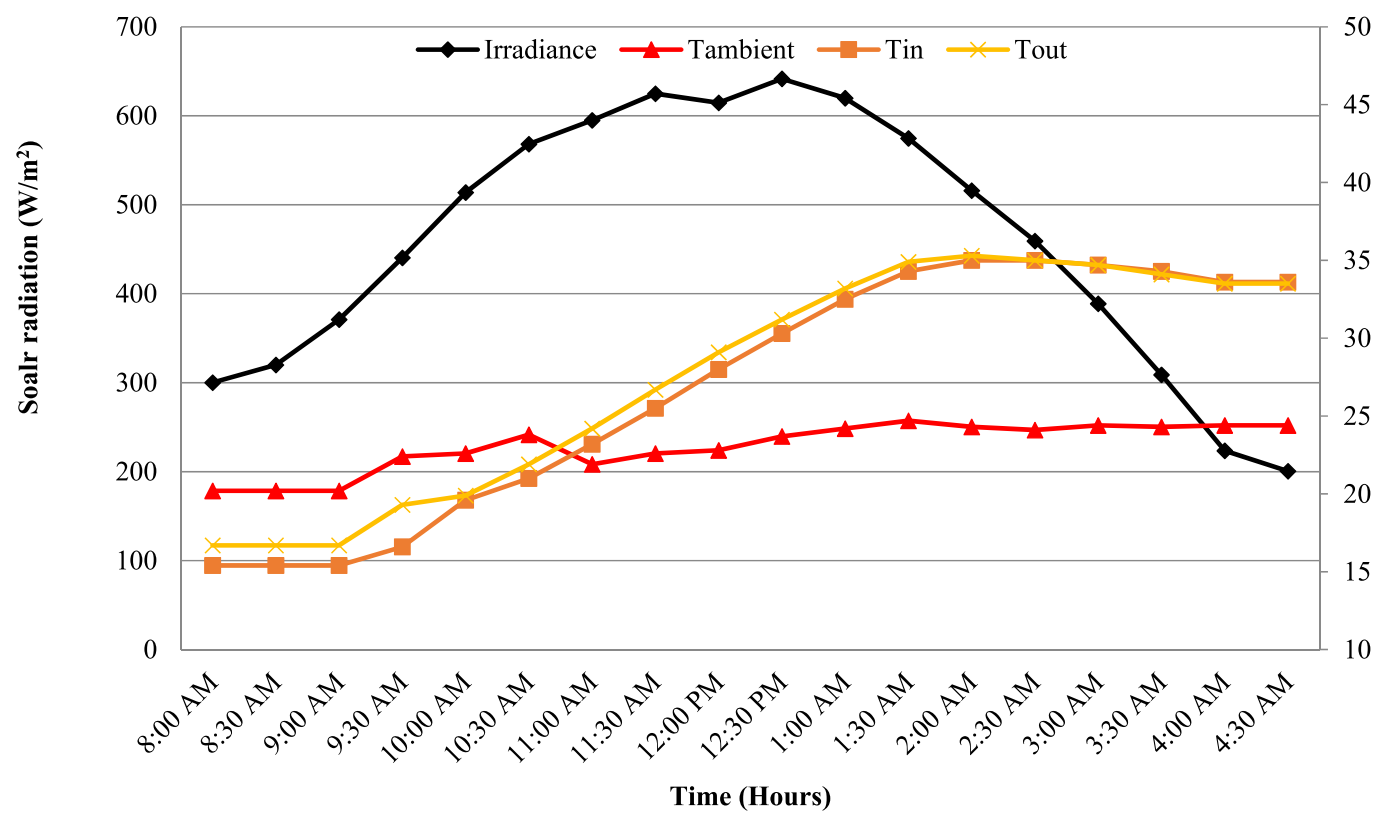

(b)

Fig. 5. (a) PV and PV/T current, voltage and power measurements; (b) Solar irradiance, ambient, inlet and outlet temperatures. 


\subsubsection{Self-organizing feature map (SOFM)}

A self-organizing feature map (SOFM) is an unsupervised artificial neural network, which is aimed to produce output with a lowdimensional by discretizing the training's input space samples (map). It utilizes a fully square-Kohonen method to determine the neighbourhoods shape as represented in Equation (7):

$$
D_{i j}=\left|X^{l}-W_{i j}\right|=\sqrt{\left(x_{1}-w_{i j 1}\right)^{2}+\ldots \ldots+\left(x_{n}-w_{i j n}\right)^{2}}
$$

where $\mathrm{X}$ is the input vector, and the weight of neighbour neurons $\mathrm{W}_{\mathrm{ij}}$.

In the training process, the network employs competitive learning between neighbouring neurons to define the winning node (best matching unit BMU), with a weight vector closely similar to the input vector as defined in Equation (8).

$D\left(k_{1}, k_{2}\right)=\min _{i, j}\left(D_{i, j}\right)$

The indexes of the winner-neuron are $\mathrm{k} 1$ and $\mathrm{k} 2$ as shown in Fig. 4(b).

Equation (9) is used to determine the neighbourhood neurons and Equation (10) is used to update the values of neuron $v$ with weight vector $\mathrm{Wv}(\mathrm{s})$.

$$
\begin{aligned}
& h(\rho, t)=\exp \left(\rho^{2} / 2 \sigma^{2}(t)\right) \\
& W v(s+1)=W v(s)+\theta(u, v, s) \cdot \alpha(s) \cdot(D(t)-W v(s))
\end{aligned}
$$

The $\rho$ is the distance from the current neuron to the BMU neuron. And $s$ is a step-index, $t$ is an index of a training sample, $u$ is the BMU-index of the input vector $\mathrm{D}(\mathrm{t})$. And the learning coefficient is $\alpha(\mathrm{s})$; the function $\theta(\mathrm{u}, \mathrm{v}, \mathrm{s})$ is the Euclidean distance of the neighbourhood of the neuron $\mathrm{v}$.

\subsubsection{Support vector machine (SVM)}

Support vector machines (SVM) is a kernel-based technique, which is nonlinear supervised learning applied in many applications, such as classification or regression. In NeuroSolutions, SVM transforms the input data into a high-dimensional map using the radial basis function (RBF), which will perform a Gaussian function on each data sample. RBF will substitute the inner product by the kernel function, which is a quadratic optimization defined in equation (11).

$$
J(\alpha)=\sum_{i=1}^{N} \alpha_{i}-\frac{1}{2} \sum_{i=1}^{N} \sum_{j=1}^{N} \alpha_{i} \alpha_{j} d_{i} d_{j} G\left(x_{i}-x_{j}, 2 \sigma^{2}\right)
$$

subject to $\sum_{i=1}^{N} d_{i} \alpha_{i}=0, \alpha_{i} \geq 0, \forall i \in\{1, \ldots, N\}$, where $\alpha_{i}$ is the starting multiplier (0.1), $\mathrm{G}$ is a Gaussian function as determined in equation (12).

$$
G\left(x, x_{i}\right)=\exp \left[\frac{1-}{2 \sigma_{i}^{2}} \sum_{1}^{p}\left(x_{k}-x_{k i}\right)^{2}\right]
$$

\section{Results and discussion}

\subsection{Experimental results}

In this study, two PV modules are implemented to measure the output power production, one is made with a thermal collector using water cooling, and the other without a thermal collector; to compare the performance of the two modules and to monitor the effect of cooling the panel on its efficiency. To evaluate the proposed PV/T system measurements have been taken for current, voltage, power, and temperatures for three continuous days from the 16th to October 18, 2020 as shown in Fig. 5 (a) and (b). From Fig. 5(a) it is found

\begin{tabular}{|c|c|c|c|c|c|c|}
\hline Statistic & S. Irradiance $\left(\mathrm{W} / \mathrm{m}^{2}\right)$ & Ambient Temperature $\left({ }^{\circ} \mathrm{C}\right)$ & PV-Current (A) & PVT-Current (A) & PVT Power (W) & PV Power (W) \\
\hline No. of observations & 435 & 435 & 435 & 435 & 435 & 435 \\
\hline Minimum & 223.60 & 17.8000 & 3.1200 & 3.1200 & 61.7760 & 63.6480 \\
\hline Maximum & 644.80 & 24.7000 & 3.2400 & 3.5400 & 70.9560 & 69.7380 \\
\hline Median & 534.50 & 22.2000 & 3.2400 & 3.3000 & 69.3360 & 66.9060 \\
\hline Mean & 505.42 & 22.2897 & 3.2166 & 3.2669 & 68.6132 & 66.7827 \\
\hline Variance (n) & 13314.93 & 1.6372 & 0.0011 & 0.0188 & 2.8461 & 2.2315 \\
\hline Standard deviation (n) & 115.39 & 1.2796 & 0.0332 & 0.1370 & 1.6870 & 1.4938 \\
\hline Standard error of the mean & 5.5389 & 0.0614 & 0.0016 & 0.0066 & 0.0810 & 0.0717 \\
\hline
\end{tabular}
that the PV/T voltage increased significantly compared with PV voltage due to the cooling of the PV panel. However, the PV current increased insignificantly compared with PV/T power for the same reason. The PV/T power is increased significantly based on the

Table 1

Descriptive statistics results of variables. 

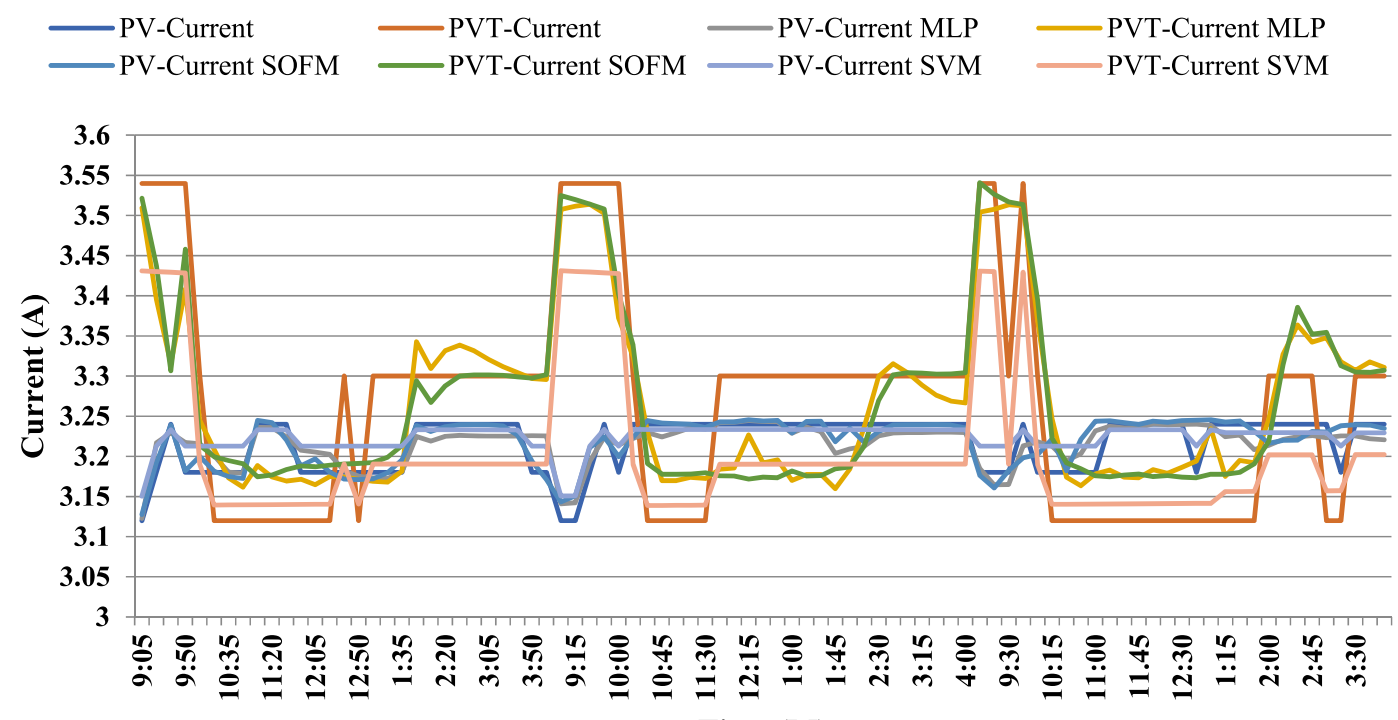

Time (M)

(a)

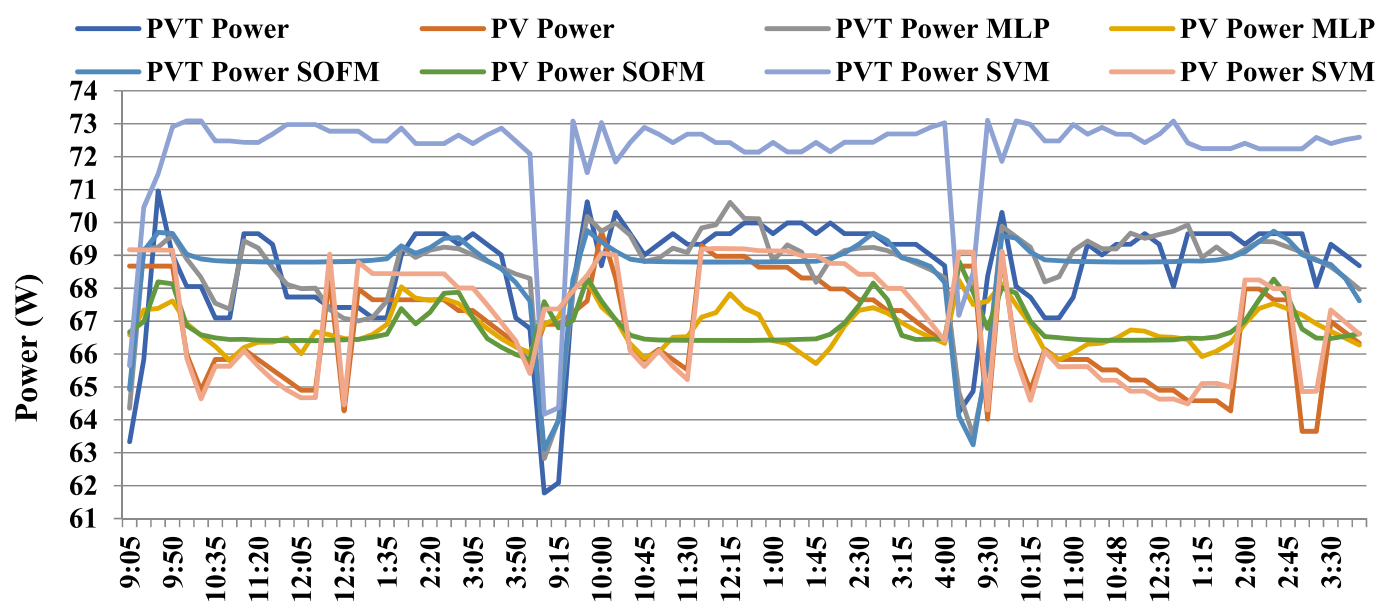

Time (M)

(b)

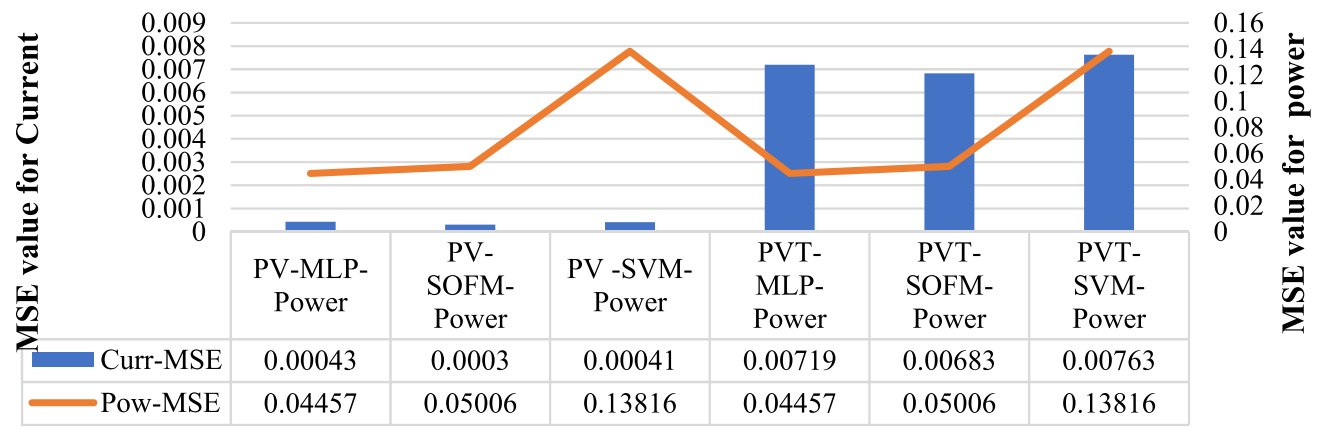

(c) 
Fig. 6. Comparison of the experimental results with the proposed systems (PV/PVT); (a) the current results; (b) the power results; (c) the Power (W) and Current (A) results based on MSE.

increment in PV/T voltage, more than the reduction in current. On the other hand, Fig. 5(b) shows the temperature and solar irradiance for the selected period. It is clear that the ambient temperature is in the range of $21-26{ }^{\circ} \mathrm{C}$ in October.

However, the solar irradiance is relatively high due to the clear sky which will reflect on PV panel temperatures. However, using a cooling observer in the PV/T system shows a clear reduction [36]. Nevertheless, the reduction in PV panel temperature is insignificant, due to the cooling weather in winter and it is expected to be more significant in summer [37,38]. It is worth mentioning that for more cooling to enhance the power production and efficiency, researchers used different observers [6], nanofluid [39], and nanophase change materials $[40,41]$. The statistical methods help to summarize, evaluate and validate the obtained output. Table 1 presents the experimental input and output variables' descriptive information using different statistical methods, such as Mean, Variance, and standard deviation (SD). It determines the positive value of the square root of the variance, which is used to examine the scope of two data sets around the Mean. A small amount of SD means closer distances around the Mean.

Table 1 shows that the two systems' output variables are closer (PV, PVT), such as PV-Current and PVT-Current, with values equal to 3.2166, 3.2669, respectively. Also, PVT Power and PV Power values are 68.6132, 66.7827, which means the power production of PVT is higher than PV Power by 2 Watt. The SD results also show slight variation and closer distances to the Mean. Besides, small Standard Errors (PV-Current $=0.0016$, PVT-Current $=0.0066$, PVT Power $=0.0810$, and PV Power $=0.0717$ ) are evidence of the Mean's reliability and the output sample's Mean accurately reflects the experiment population Means.

\subsection{ANN design and results}

The experiments undertaken were tested proposed systems under the same conditions to measure each system's efficiency separately to compare it with the presented development results. The Neurosolutions package is used to deploy three neural networks implemented in this paper to calculate and predict the renewable energy power generated by the photovoltaics (PV) system. The experiments trained MLP, SOFM, and SVM using about 450 datasets. The datasets are divided into $50 \%$ as input data, $20 \%$ as crossvalidation data, and $30 \%$ as testing data. All the networks consist of 1 input layer, 1 hidden layer, and 1 output layer. The input variables are Solar irradiance $\left(\mathrm{W} / \mathrm{m}^{2}\right)$, Ambient Temperature $\left({ }^{\circ} \mathrm{C}\right)$, and the output variables are PV-Current (A), PVT-Current (A), PVT Power (W), PV Power (W). The maximum number of epochs is 1000 . The TanhAxon with a bias and Tanh formula is used as a transfer function in the hidden and output layers. The momentum rule is used as a learning rule with a rate of 0.7. The backpropagation algorithm (BP) is used to determine the network's errors to adjustable processing elements' weights. Fig. 6(a) presents the proposed neural models' comparison results (MLP, SOFM, and SVM) for both PV and PVT systems with the experiment's current desired datasets. It shows close fits, which means that the proposed neural models simulate the real data behaviour and easily forecast the data's future figure. Fig. 6(b) shows the proposed neural models' comparison results (MLP, SOFM, and SVM) for PV and PVT systems with the experiment's power desired datasets. It confers close fits of the proposed neural models' output with the real data, which could help forecast the data's future figure.

Table 2 demonstrates the proposed models' results based on the validation factors, such as MSE, NMSE, MAE, and correlation rate R. The three proposed models (MLP, SOFM, and SVM) achieved excellent MSE results for generating the current values of the PV system $(0.00043,0.00030,0.00041)$ and PVT system $(0.00719,0.00683,0.00763)$, respectively, as shown in Fig. 6 (c). Additionally, they obtained excellent results of MAE for the three proposed models.

Fig. 7 presents the Comparison results of (NMSE, R) for the two proposed systems (PV/PVT) based on Current and Power experimental datasets. The correlation rate of (PV-MLP and PV-SOFM) of the power results $(0.467,0.417)$ is lower than other neural methods, such as PV-SVM that achieves 0.971 .

Table 3 presents the proposed models' results validation factors, such as MSE, NMSE, MAE, and correlation rate r. The proposed models (MLP, SOFM, and SVM) delivered excellent MSE results for simulating the power values of the PV system (0.04457, 0.05006, $0.13816)$ and PVT system $(0.04457,0.05006,0.13816)$, respectively. Additionally, they achieved remarkable results of MAE for the three proposed models.

Finally, the proposed neural models are able to generate future figures for any needed period of time. Whilst testing the ANN models' generalization, we generated data for three days to compare with actual data sets. Fig. 8 illustrates the experimental datasets of PV and PVT systems' power and the generated (Forecasting) datasets. The figure shows a close fit between the actual and predicated datasets.

Table 2

Proposed models' current results validation factors.

\begin{tabular}{lllllc}
\hline Performance & PV-MLP-Current & PV-SOFM-Current & PV-SVM-Current & PVT-MLP-Current & PVT-SOFM-Current \\
\hline MSE & 0.00043 & 0.00030 & 0.00041 & 0.00719 & 0.00683 \\
NMSE & 0.39048 & 0.27815 & 0.37695 & 0.38329 & 0.36425 \\
MAE & 0.01622 & 0.01087 & 0.01646 & 0.06749 & 0.00763 \\
Min Abs Error & 0.00017 & 0.00018 & 0.00650 & 0.00055 & 0.4066 \\
R & 0.78224 & 0.852420 & 0.91697 & 0.78546 & 0.00011 \\
\hline
\end{tabular}




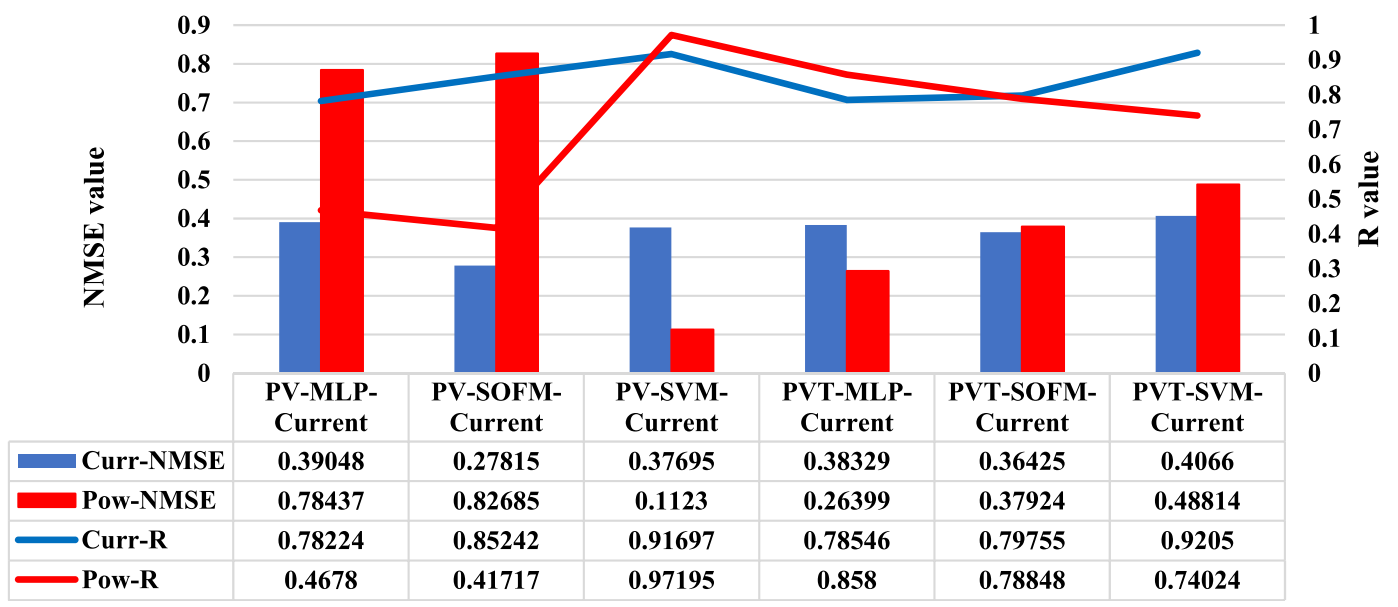

Fig. 7. Comparison of Current (A) and Power (W) results based on (NMSE, R) of the two proposed systems (PV/PVT).

Table 3

Proposed models' power results validation factors.

\begin{tabular}{lllllll}
\hline Performance & PV-MLP-Power & PV-SOFM-Power & PV -SVM-Power & PVT-MLP-Power & PVT-SOFM-Power & PVT-SVM- Power \\
\hline MSE & 0.04457 & 0.05006 & 0.13816 & 0.04457 & 0.05006 & 0.13816 \\
NMSE & 0.78437 & 0.82685 & 0.11230 & 0.26399 & 0.37924 & 0.48814 \\
MAE & 1.00022 & 1.09567 & 0.42700 & 0.65084 & 0.87393 & 3.53054 \\
Min Abs Error & 0.00098 & 0.08092 & 0.04294 & 0.01337 & 0.01522 & 0.51574 \\
R & 0.46780 & 0.41717 & 0.97195 & 0.85800 & 0.78848 & 0.74024 \\
\hline
\end{tabular}

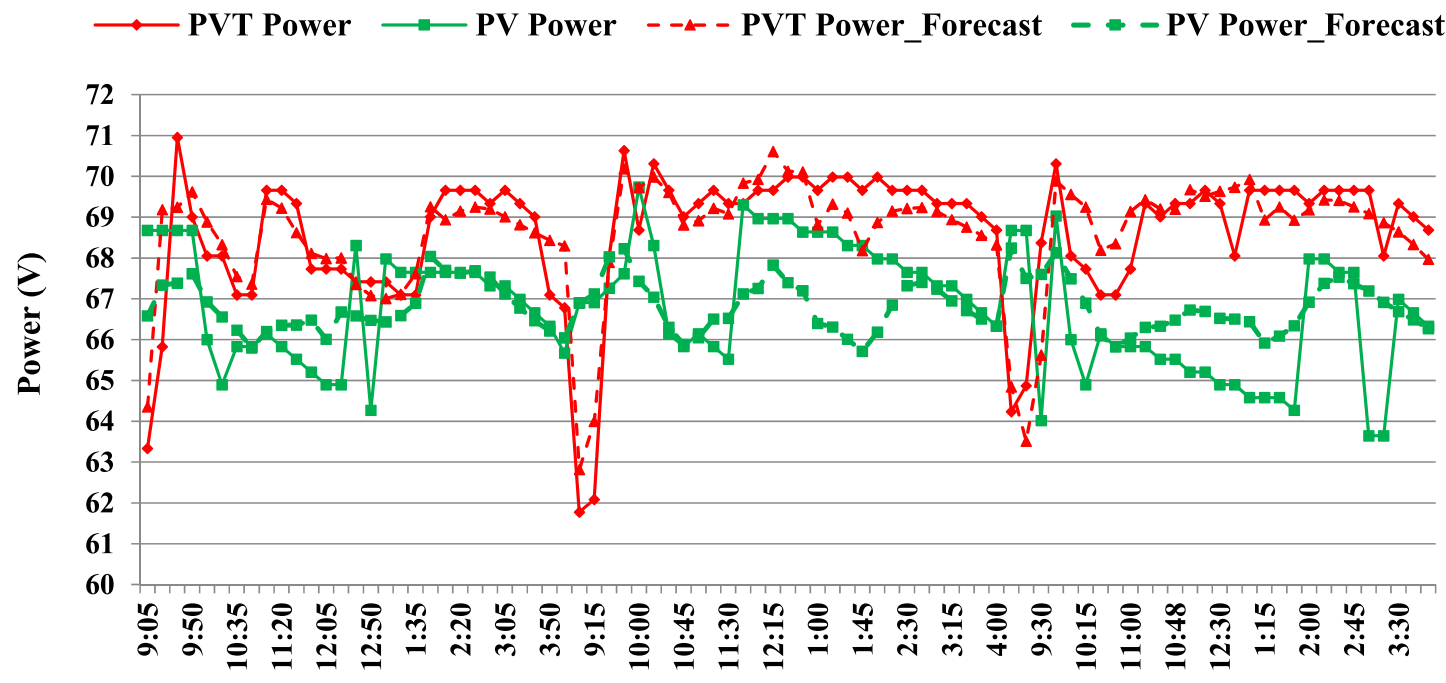

Time (M)

Fig. 8. The experimental datasets of PV and PVT systems' power and the generated (Forecasting) datasets.

\section{Conclusion}

This paper discussed the factors affecting the production of solar modules in hot weather conditions such as in the Sohar/Sultanate of Oman. some of the most important factors are the location of the photovoltaic system, the ambient temperature, the strength of solar irradiance, humidity, the proportion of dust accumulated on the panels etc. Among the most important contributions to the current work is creating efficient mathematical models that easily apply and simulate photovoltaic cell production and accurately predict future production. This helps to calculate the amount of electrical energy production, as well as its requirements and cost. Furthermore, the proposed mathematical models could accurately analyze the economic cost of the photovoltaic energy system and the future 
cost of producing any quantities of energy. The in-depth analysis of previous studies and research has led to the discovery of gaps that must be addressed in the proposed models, the most important of which is accurate and easy production prediction for any period. Also, the ability to identify the most important factors affecting photovoltaic energy production correctly and quickly. Most of the past models lacked an accurate comparison between the results recorded and the results from mathematical models due to the inability to apply them under the same conditions. At the same time, the current work was able to measure the data in the North Al Batinah region/ Sultanate of Oman to compare it with the production of the proposed mathematical models under the same conditions. The current work aims to predict and evaluate a thermal PV system using artificial neural network models based on an experimental data set in Oman. A PVT and conventional PV has been installed in Sohar with weather station, sensors and data acquisition to measure electrical quantities and weather data. It is found that PVT and PV power values are 68.6132, and 66.7827, respectively, which means the power production of PVT is higher than PV Power by 2 Watt. The results demonstrate that the three proposed models (MLP, SOFM, and SVM) achieved excellent MSE results for generating current values for the PV system $(0.00043,0.00030,0.00041)$ and PVT system $(0.00719$, $0.00683,0.00763)$, respectively. The proposed models also provided excellent MSE results for simulating the energy values of the PV system $(0.04457,0.05006,0.13816)$ and the PVT system $(0.04457,0.05006,0.13816)$, respectively. Finally, the proposed neural models can generate future numbers for any desired interval that fit neatly into actual data sets.

\section{Author statement}

JHY: Conceptualization; Formal analysis; Funding acquisition; Investigation; Methodology; Project administration; Software; Supervision; Validation; Visualization; Roles/Writing - original draft; Writing - review \& editing. HKA: Data curation; Formal analysis; Resources; Software; Supervision; Validation; Visualization; Roles/Writing - original draft; Writing - review \& editing.

\section{Declaration of competing interest}

"We the authors declare no affiliations with or involvement in any organization or entity with any financial interest (such as honoraria; educational grants; participation in speakers' bureaus; membership, employment, consultancies, stock ownership, or other equity interest; and expert testimony or patent licensing arrangements), or non-financial interest (such as personal or professional relationships, affiliations, knowledge or beliefs) in the subject matter or materials discussed in this manuscript".

\section{Acknowledgment}

"The research leading to these results has received a research project grant funding from the Research Council of the Sultanate of Oman, Research Grant Agreement No. SU/BFP/RG/03".

\section{References}

[1] A.H. Al-Waeli, H.A. Kazem, J.H. Yousif, M.T. Chaichan, K. Sopian, Mathematical and neural network models for predicting the electrical performance of a PV/T system, Int. J. Energy Res. 43 (14) (2019) 8100-8117.

[2] M.T. C, K. S, Ali H.A. Alwaeli, Hussein A. Kazem, Photovoltaic/Thermal ( PV/T ) Systems: Principles, Design, and Applications, first ed., Springer Nature, Switzerland, 2019.

[3] J.H. Yousif, H.A. Kazem, N.N. Alattar, I.I. Elhassan, A comparison study based on artificial neural network for assessing PV/T solar energy production, Case Stud. Therm. Eng. 13 (2019) 100407. January.

[4] H.A. Kazem, M.T. Chaichan, A.H.A. Al waeli, K. Sopian, Evaluation of aging and performance of grid-connected photovoltaic system northern Oman, Seven years ' experimental study," 207 (2020) 1247-1258. April.

[5] J.H. Yousif, H.A. Kazem, J. Boland, "Predictive models for photovoltaic electricity production in hotweather conditions, Energies 10 (7) (2017), https://doi.org/ 10.3390/en10070971 p.971.

[6] J.H. Yousif, H.A. Al-Balushi, H.A. Kazem, M.T. Chaichan, Analysis and forecasting of weather conditions in Oman for renewable energy applications, Case Studies in Thermal Engineering 13 (2019) p.100355.

[7] J.H. Yousif, H.A. Kazem, Modeling of daily solar energy system prediction using soft computing methods for Oman. Research Journal of Applied Sciences, Eng. Technol. 13 (3) (2016) 237-244.

[8] K. Al-Waeli, H. A, H.A. Kazem, M.T. Chaichan, Sopian, A Review of Photovoltaic Thermal Systems : Achievements and Applications, 2020, pp. 1-40. April.

[9] H.A. Kazem, J.H. Yousif, "Comparison of prediction methods of photovoltaic power system production using a measured dataset," Energy Convers, OR Manag. 148 (2017) 1070-1081.

[10] A.H.A. Al Waeli, H.A. Kazem, J.H. Yousif, M.T. Chaichan, K. Sopian, "Mathematical and neural network modeling for predicting and analyzing of nanofluidnano PCM photovoltaic thermal systems performance," Renew, Energy 145 (2020) 963-980.

[11] A. Saberian, H. Hizam, M.A.M. Radzi, M.Z.A. Ab Kadir, M. Mirzaei, Modelling and prediction of photovoltaic power output using artificial neural networks, International Journal of Photoenergy 2014 (2014) 1-10, https://doi.org/10.1155/2014/469701, 469701.

[12] A. Ghanbarzadeh, A.R. Noghrehabadi, E. Assareh, M.A. Behrang, Solar radiation forecasting based on meteorological data using artificial neural networks, IEEE Int. Conf. Ind. Informatics (2009) 227-231.

[13] M.A. Behrang, E. Assareh, A. Ghanbarzadeh, A.R. Noghrehabadi, The potential of different artificial neural network (ANN) techniques in daily global solar radiation modeling based on meteorological data, Sol. Energy 84 (8) (2010) 1468-1480.

[14] S. Rehman, M. Mohandes, Artificial neural network estimation of global solar radiation using air temperature and relative humidity, Energy Pol. 36 (2) (2008) $571-576$.

[15] A. Mellit, A.M. Pavan, A 24-h forecast of solar irradiance using artificial neural network: application for performance prediction of a grid-connected PV plant at Trieste, Italy, Sol. Energy 84 (5) (2010) 807-821.

[16] S.F.Z. Asl, A. Karami, G. Ashari, A. Behrang, A. Assareh, N. Hedayat, "Daily global solar radiation modeling using multi-layer perceptron (MLP) neural networks, World Acad. Sci. Eng. Technol 79 (7) (2011) 740-742.

[17] M.H. Al Shamisi, A. H, H.A.N. Hejase, "Using MATLAB to Develop artificial neural network models for predicting global solar radiation in Al ain city - UAE, Eng. Educ. Res. Using MATLAB (2011). 
[18] J. Qin, Z. Chen, K. Yang, S. Liang, W. Tang, "Estimation of monthly-mean daily global solar radiation based on MODIS and TRMM products, Appl. Energy 88 (7) (2011) 2480-2489.

[19] A. Hasni, A. Sehli, B. Draoui, A. Bassou, B. Amieur, Estimating global solar radiation using artificial neural network and climate data in the south-western region of Algeria, Energy Procedia 18 (2012) 531-537.

[20] T. Khatib, A. Mohamed, M. Mahmoud, Estimating global solar energy using multilayer perception artificial neural network, Int. J. Energy 6 (1) (2012) 25-33.

[21] G. Salima, G.M.S. Chavula, Determining angstrom constants for estimating solar radiation in Malawi, Int. J. Geosci. 3 (2) (2012) 391-397.

[22] M. Ben Ammar, M. Neji, A.M. Alimi, G. Gouardères, The affective tutoring system, Expert Syst. Appl. 37 (4) (2010) $3013-3023$.

[23] A.H. Assi, M.H. Al-Shamisi, H.A.N. Hejase, A. Haddad, Prediction of global solar radiation in UAE using artificial neural networks, in: Proc. 2013 Int. Conf. Renew. Energy Res. Appl. ICRERA 2013, 2013, pp. 196-200. October.

[24] Y. Jiang, Computation of monthly mean daily global solar radiation in China using artificial neural networks and comparison with other empirical models, Energy 34 (9) (2009) 1276-1283.

[25] R. Yacef, A. Mellit, S. Belaid, Z. Şen, New combined models for estimating daily global solar radiation from measured air temperature in semi-arid climates: application in Ghardaïa, Algeria, Energy Convers. Manag. 79 (2014) 606-615.

[26] K. Suryanarayana Rao, R.O. Recknagel, Early onset of lipoperoxidation in rat liver after carbon tetrachloride administration, Exp. Mol. Pathol. 9 (2) (1968) $271-278$.

[27] E.A. Ahmed, M.E.-N. Adam, Estimate of global solar radiation by using artificial neural network in Qena, upper Egypt, J. Clean Energy Technol 1 (2) (2013) $148-150$.

[28] X. Yang, F. Jiang, H. Liu, Short-term power prediction of photovoltaic plant based on SVM with similar data and wavelet analysis, Przeglad Elektrotechniczny 89 (5) (2013) 81-85.

[29] M.A. Hamdan, S. Maheshwari, J.T. Fahey, W.E. Hellenbrand, Endovascular stents for coarctation of the aorta: initial results and intermediate-term follow-up, J. Am. Coll. Cardiol. 38 (5) (2001) 1518-1523.

[30] S. Benkaciali, M. Haddadi, A. Khellaf, K. Gairaa, M. Guermoui, Evaluation of the global solar irradiation from the artificial neural network technique, Rev. des Energies Renouvelables 19 (4) (2016) 617-631.

[31] F. Ghani, M. Duke, J.K. Carson, Estimation of photovoltaic conversion efficiency of a building integrated photovoltaic/thermal (BIPV/T) collector array using an artificial neural network, Sol. Energy 86 (11) (2012) 3378-3387.

[32] A. Ahmad, T. Anderson, Global solar radiation prediction using artificial neural network models for New Zealand, in: 52nd Annu. Conf. Aust. Sol. Counc., 2014, pp. 141-150. May.

[33] H.A. Kazem, M.T. Chaichan, The effect of dust accumulation and cleaning methods on PV panels' outcomes based on an experimental study of six locations in Northern Oman, Sol. Energy 187 (2019) 30-38. January.

[34] Hussein A. Kazem, Jabar Yousif, Miqdam T. Chaichan, Ali HA. Al-Waeli, Experimental and deep learning artificial neural network approach for evaluating gridconnected photovoltaic systems, Int. J. Energy Res. 43 (14) (2019) 8572-8591, https://doi.org/10.1002/er.4855.

[35] Al-Waeli, Ali Ha, K. Sopian, H. Jabar, Yousif, A. Hussein, John Kazem, Boland, Miqdam T. Chaichan, Artificial neural network modeling and analysis of photovoltaic/thermal system based on the experimental study, Energy Convers. Manag. 186 (2019) 368-379, https://doi.org/10.1016/j. enconman.2019.02.066.

[36] A.H. Al-Waeli, H.A. Kazem, K. Sopian, M.T. Chaichan, Techno-economical assessment of grid connected PV/T using nanoparticles and water as base-fluid systems in Malaysia, Int. J. Sustain. Energy 37 (6) (2018) 558-575.

[37] M. Sangeetha, S. Manigandan, M.T. Chaichan, V. Kumar, Progress of MWCNT, Al2O3, and CuO with water in enhancing the photovoltaic thermal system, Int. J. Energy Res. 44 (2) (2020) 821-832.

[38] H.A. Kazem, A.H. Al-Waeli, M.T. Chaichan, K.H. Al-Waeli, A.B. Al-Aasam, K. Sopian, Evaluation and comparison of different flow configurations PVT systems in Oman: a numerical and experimental investigation, Sol. Energy 208 (2020) 58-88.

[39] A.H.A. Al-Waeli, K. Sopian, M.T. Chaichan, H.A. Kazem, H.A. Hasan, A.N. Al-Shamani, An experimental investigation of SiC nanofluid as a base-fluid for a photovoltaic thermal PV/T system, Energy Convers. Manag. 142 (2017).

[40] Al-Waeli, K. Ali Ha, Sopian, Hussein A. Kazem, Jabar H. Yousif, Miqdam T. Chaichan, Adnan Ibrahim, Sohif Mat, Mohd Hafidz Ruslan, Comparison of prediction methods of PV/T nanofluid and nano-PCM system using a measured dataset and artificial neural network, Sol. Energy 162 (2018) $378-396$.

[41] A.H. Al-Waeli, M.T. Chaichan, K. Sopian, H.A. Kazem, Influence of the base fluid on the thermo-physical properties of PV/T nanofluids with surfactant, Case Studies in Thermal Engineering 13 (2019) 100340. 